\title{
Examination of individual and multiple comorbid conditions and health-related quality of life in older cancer survivors
}

\author{
Elizabeth J. Siembida ${ }^{1,2,5}$ ( Ashley Wilder Smith ${ }^{3} \cdot$ Arnold L. Potosky $^{4} \cdot$ Kristi D. Graves $^{4} \cdot$ Roxanne E. Jensen $^{3}$
}

Accepted: 15 November 2020 / Published online: 14 January 2021

(C) This is a U.S. Government work and not under copyright protection in the US; foreign copyright protection may apply 2021

\begin{abstract}
Purpose Older cancer survivors ( $\geq 65$ years at diagnosis) are at high-risk for multimorbidity $(2+$ comorbid conditions). However, few studies have utilized a generalizable sample of older cancer survivors to understand how individual comorbid conditions, as opposed to total comorbidity burden, are associated with health-related quality of life (HRQOL). We examined associations between HRQOL outcomes (pain, fatigue, physical function), individual comorbidities (cardiovascular disease [CVD], lung disease, diabetes, arthritis) and total comorbidity (cancer-only, cancer +1 condition, cancer +2 or more conditions).

Methods Utilizing a population-based sample of 2019 older cancer survivors, we tested associations between comorbid conditions and the HRQOL outcomes using generalized linear models. HRQOL domains were assessed using Patient-Reported Outcome Measurement Information System ${ }^{\circledR}\left(\right.$ PROMIS $\left.^{\circledR}\right)$ measures. Comorbidity was assessed via self-report.

Results Cancer survivors with lung disease reported significantly worse physical functioning $(\beta=-4.96, p<0.001)$, survivors with arthritis reported significantly higher pain $(\beta=4.37, p<0.001)$, and survivors with CVD reported significantly higher fatigue $(\beta=3.45, p<0.001)$ compared to survivors without each condition. Having cancer +1 condition was not as strongly associated with all outcomes as when individual conditions were tested (e.g. pain: $\beta=3.09, p<0.001$ ). Having $2+$ comorbidities had a stronger association with all outcomes (e.g. physical function: $\beta=-7.51, p<0.001$ ) than examining conditions individually.

Conclusions Knowing the specific comorbid condition profile of an older cancer survivor provides insight into specific HRQOL outcomes that may be impaired in cancer survivorship, but understanding total comorbidity burden, regardless of the specific conditions, sheds light on survivors at-risk for multiple impairments in HRQOL. This information, taken together, can inform risk-stratified survivorship care.
\end{abstract}

Keywords Symptoms $\cdot$ Cancer $\cdot$ Comorbidity $\cdot$ Older adults $\cdot$ Health-related quality of life

\section{Plain English summary}

Disclaimer: This article was prepared as part of two of the author's (REJ, AWS) official duties as employees of the US Federal Government, and as part of one author's (EJS) postdoctoral fellowship with a US Federal Government institution (the National Cancer Institute). The findings and conclusions in this report are those of the authors and do not necessarily represent the official position of the National Cancer Institute.

Electronic supplementary material The online version of this article (https://doi.org/10.1007/s11136-020-02713-0) contains supplementary material, which is available to authorized users.

Elizabeth J. Siembida

esiembida@northwell.edu

Extended author information available on the last page of the article
Older cancer survivors ( $\geq 65$ years at diagnosis) are often managing multiple chronic health conditions (e.g. heart disease, diabetes) while undergoing and after cancer treatment. Previous research has most often examined how the total number of chronic conditions influences health-related quality of life (HRQOL), but few studies have explored whether specific chronic conditions influence specific areas of HRQOL, like pain. We tested the association between HRQOL domains (pain, fatigue, physical function) and individual comorbid conditions (heart disease, lung disease, diabetes, arthritis) in a sample of older cancer survivors. We also tested the association between the same three HRQOL domains and the total number of comorbid 
conditions. Our results found that when examining only one additional chronic condition, knowing the specific condition (e.g. heart disease) has a larger influence on HRQOL than when examining the total count of conditions. However, if a survivor has two or more chronic health conditions, they are at increased risk for poor HRQOL in all the domains we examined, regardless of the specific chronic condition. The results of this study provide useful information for risk-stratified survivorship care and identifying older cancer survivors who will require tailored and timely symptom management.

\section{Introduction}

Approximately $67 \%$ of older adults (age $\geq 65$ ) in the USA have multimorbidity ( $2+$ co-occurring comorbid conditions) [1], and the risk for multimorbidity is higher among older cancer survivors $[2,3]$. Both cancer treatment and common comorbid conditions (e.g. cardiovascular disease [CVD]) can impact an older survivor's symptom burden and overall health-related quality of life (HRQOL) [4-6]. Pain, fatigue, and physical function limitations are some of the most commonly impaired domains of HRQOL reported by older cancer survivors [7-11]. Understanding how multimorbidity may influence older cancer survivors' outcomes following diagnosis is an important step in identifying and tailoring symptom management for survivors.

Multimorbidity is commonly captured as a numerical count or as a comorbidity index (e.g. Charlson Comorbidity Index, NCI Comorbid Index). These indices were developed and weighted to assess the role of comorbidity on overall survival [12-15], not HRQOL, and therefore may not appropriately weight conditions likely to impact HRQOL. Identification of the specific relationships between types of comorbidities and HRQOL provide a window into the survivors most at-risk for problematic outcomes after cancer treatment, and can assist with providing more specific recommendations to heath care providers who care for older cancer survivors.

Research involving cancer survivors with multimorbidity has shown variable relationships between multimorbidity and cancer outcomes including overall survival [16-22], treatment complications [23, 24], and lower likelihood of guideline-concordant care [25-27]. Some studies also suggest significantly lower HRQOL among multimorbid survivors [28-30], but few studies have explored individual comorbidities and their associations with specific HRQOL outcomes.

In this study we aimed to tease out the differences in HRQOL when examining comorbid conditions both separately and as a count variable. We examined associations between CVD, lung disease, diabetes, arthritis and three common HRQOL outcomes (pain, fatigue, physical function) in a population-based sample of older cancer survivors 6-12 months postdiagnosis. We also tested the association between a comorbid count variable (cancer-only, cancer +1 condition, cancer +2 or more conditions) and pain, fatigue, and physical function to determine if the conclusions differ when looking at the independent association of each comorbid condition compared to a categorical count of the total number of conditions.

\section{Methods}

\section{Data collection and study population}

The current study used data from the population-based Measuring Your Health (MY-Health) study. The MYHealth study design and methods have been described in detail elsewhere [31]. Briefly, 15,300 individuals diagnosed with cancer between 2010 and 2012 were contacted by four Surveillance, Epidemiology, and End Results (SEER) cancer registries (California [2], Louisiana, and New Jersey). A total of 5506 individuals completed a self-report, mailbased survey for a response rate of $36 \%$. We used the following inclusion criteria: (1) completed the baseline MYHealth survey between 6 and 12 months from diagnosis ( $N=162$ excluded), (2) were diagnosed with their first cancer (prostate, colorectal, non-small cell lung, Non-Hodgkin lymphoma, female breast, uterine, or cervical) at the age of 65 years or older ( $N=3240$ excluded), and (3) had Stage I-IV cancer based on the derived AJCC 7th edition staging criteria ( $N=85$ excluded). The final sample was $N=2019$. The research design and study methods for the MY-Health study were approved by the Institutional Review Board at Georgetown University.

\section{Measures}

\section{Health-related quality of life}

In this study, we examined three HRQOL domains (pain, fatigue, and physical function) using Patient Reported Outcomes Measurement Information System ${ }^{\circledR}$ (PROMIS ${ }^{\circledR}$ ) tailored short forms [31]. Pain interference was measured using 11 items selected from the Pain Interference PROMIS item bank v1.0 (Cronbach's alpha $[\alpha]=0.91$ ), fatigue with 14 items selected from the Fatigue PROMIS item bank v1.0 $(\alpha=0.89)$, and physical function with 16 items selected from the Physical Function PROMIS item bank v1.0 $(\alpha=0.92)$. All PROMIS short forms were scored using a T-score metric with a mean of 50 and standard deviation (SD) of 10 and were normalized to the general US population, meaning that 
a score of 50 represents the average US adult's experience of the HRQOL domain of interest [32]. For pain and fatigue, higher scores represent greater symptom burden. For physical functioning, higher scores represent better functioning.

\section{Multimorbidity}

Patients self-reported their history of ten comorbid conditions: (1) heart attack; (2) heart failure or congestive heart failure; (3) stroke; (4) asthma; (5) lung disease; (6) diabetes; (7) arthritis; (8) depression; (9) anxiety; and (10) a sleep disorder. For each condition, survivors were categorized as having the condition (responding 'Yes' to survey item) or not having the condition (responding 'No,' 'Unsure,' or missing survey item). We focused on four of these comorbidities (CVD, lung disease, diabetes, arthritis) common among older adults (hereafter 'selected conditions') [1]. Survivors were coded as having a history of CVD if they answered 'yes' to either heart attack and/or heart failure.

To control for the presence of other comorbid conditions in our analyses and examine the independent effect of the selected conditions, we created an additional comorbid conditions indicator using the 10 comorbidities identified in the survey (excluding the condition being independently examined in the model). Other conditions were coded as either $0=$ no additional condition or $1=$ one or more additional comorbid conditions. Finally, we created a comorbid count variable (cancer-only, cancer +1 condition, cancer +2 or more conditions) using all 10 self-reported comorbid conditions.

\section{Sociodemographic variables}

Sociodemographic characteristics were obtained from self-report data and through SEER. Self-report variables included: education attainment ( $<$ high school graduate, high school graduate or GED/some college, $\geq$ college degree), race/ethnicity (Non-Hispanic White, Non-Hispanic Black, Hispanic, Non-Hispanic Asian, Other race/ethnicity/multiracial), and marital status (married/living with a partner, never married/separated/divorced/widowed). We obtained data on survivors' sex (male/female) from SEER.

\section{Cancer clinical variables}

Survivors' clinical characteristics were obtained through self-report and SEER. SEER data included: cancer site (lung cancer, breast cancer, gynecologic cancer [uterine, cervical], colorectal cancer, non-Hodgkin lymphoma, prostate cancer), stage (as measured by American Joint Committee on Cancer, $7^{\text {th }}$ edition: early stage [I/II], advanced stage [III/IV]), and age at diagnosis. We combined uterine and cervical cancer into one 'gynecologic cancer' category due to small sample size. Cancer treatment information was collected via selfreport. Older cancer survivors were asked, separately, if they ever received chemotherapy [yes/no], radiation [yes/ no], and/or surgery [yes/no] as part of their cancer treatment.

\section{Statistical analysis}

We computed descriptive statistics for sociodemographic, clinical, and outcome variables. We created generalized linear models for each selected condition and each outcome measure (pain, fatigue, physical function). Each generalized linear model included self-reported history of the selected condition as the primary independent variable, and was adjusted for sociodemographic characteristics, clinical characteristics, and presence of other comorbid conditions. We also tested a generalized linear model for each outcome (pain, fatigue, physical function) using the comorbid count variable as the primary independent variable. The fully adjusted models controlled for sociodemographic and clinical characteristics. To identify possible differences in the associations between comorbid count and each outcome across cancer type, we also conducted a sensitivity analysis that stratified the comorbid count models by the four most common cancer types in our sample (lung cancer, colorectal cancer, breast cancer, and prostate cancer). We were unable to conduct these sensitivity analyses for the selected conditions models because of small sample sizes.

Previous research in cancer survivors has identified ranges of PROMIS T-scores that indicate minimally important differences between groups for fatigue (2.5-5.0 points), pain interference (4.0-6.0 points), and physical function (4.0-6.0 points) [33]. Minimally important differences have been used in previous work with PROMIS data to suggest clinically meaningful results, and we examine differences between survivors with and without the four selected conditions using the lower bound of these ranges as the threshold for clinically meaningful results. All tests were two-sided, the significance level was set at 0.001 to account for the multiple comparisons, and analyses were completed using SAS 9.4 (Cary, NC).

\section{Results}

We found that $74 \%(N=1494)$ of cancer survivors were multimorbid (at least 1 additional comorbidity), and just under half had 2 or more comorbid conditions (45.7\%; Table 1). Overall, cancer survivors reported physical function limitations notably below the US population average of 50 (Mean $[M]=43.7$, Standard Deviation $[\mathrm{SD}]=9.8)$ [32]. In contrast, the overall sample reported pain $(M=51.8, \mathrm{SD}=10.4)$ and 
Table 1 Sociodemographic and clinical characteristics of older cancer survivors $(N=2019)$

\begin{tabular}{|c|c|}
\hline & $N(\%)$ \\
\hline Age at diagnosis (mean, standard deviation) & $71.8(4.97)$ \\
\hline \multicolumn{2}{|l|}{ Sex } \\
\hline Male & $1029(51.0)$ \\
\hline Female & $990(49.0)$ \\
\hline \multicolumn{2}{|l|}{ Marital status } \\
\hline Married/living with a partner & $1246(62.3)$ \\
\hline Never married/separated/divorced/widowed & $755(37.7)$ \\
\hline \multicolumn{2}{|l|}{ Education } \\
\hline Less than high school grad & $436(21.9)$ \\
\hline High school grad or completed some college & $1028(51.6)$ \\
\hline Completed a college degree or higher & $527(26.5)$ \\
\hline \multicolumn{2}{|l|}{ Race/ethnicity } \\
\hline Non-Hispanic White & $985(48.8)$ \\
\hline Non-Hispanic Black & $358(17.7)$ \\
\hline Hispanic & $333(16.5)$ \\
\hline Non-Hispanic Asian & $288(14.3)$ \\
\hline Other race/multiple races & $55(2.7)$ \\
\hline \multicolumn{2}{|l|}{ Cancer site } \\
\hline Lung cancer & $372(18.4)$ \\
\hline Breast cancer & $414(20.5)$ \\
\hline Gynecologic cancer & $115(5.7)$ \\
\hline Colorectal cancer & $380(18.8)$ \\
\hline Non-Hodgkin lymphoma & $178(8.8)$ \\
\hline Prostate cancer & $560(27.7)$ \\
\hline \multicolumn{2}{|l|}{ Stage of disease } \\
\hline Stage I/II & $1441(71.4)$ \\
\hline Stage III/IV & $578(28.6)$ \\
\hline \multicolumn{2}{|l|}{ History of chemotherapy } \\
\hline Received chemotherapy & $782(39.8)$ \\
\hline Did not receive chemotherapy & $1183(60.2)$ \\
\hline \multicolumn{2}{|l|}{ History of radiation } \\
\hline Received radiation & $830(41.9)$ \\
\hline Did not receive radiation & $1151(58.1)$ \\
\hline \multicolumn{2}{|l|}{ Comorbid conditions } \\
\hline Cardiovascular disease & $264(13.1)$ \\
\hline Arthritis & $852(42.2)$ \\
\hline Diabetes & 577 (28.6) \\
\hline Lung disease & $312(15.5)$ \\
\hline \multicolumn{2}{|l|}{ Total number of comorbid conditions } \\
\hline Cancer only & $525(26.0)$ \\
\hline 1 & $571(28.3)$ \\
\hline 2 & $431(21.4)$ \\
\hline 3 & $246(12.2)$ \\
\hline 4 or more & $246(12.3)$ \\
\hline
\end{tabular}

fatigue levels $(M=51.0, S D=10.5)$ similar to the general population. When examining the characteristics of survivors with each of the four selected conditions, we saw differences

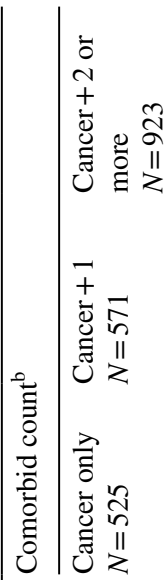

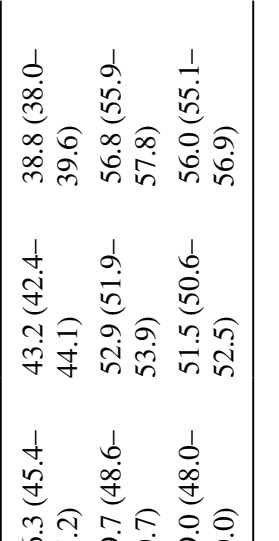

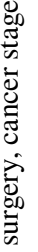

守守守它宗官

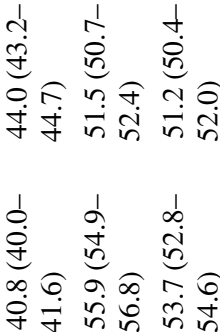

西

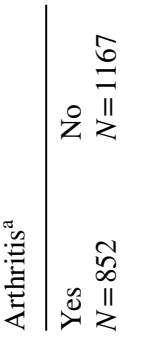

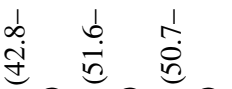

ที่

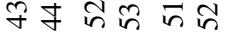

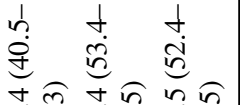

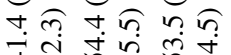

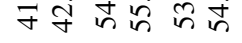

$\begin{array}{lll}1 & 1 & 1 \\ & n & 0\end{array}$

m

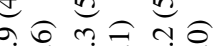

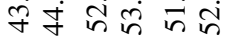

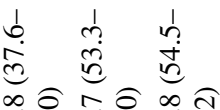

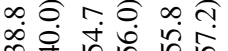

$\approx z$ 
in sociodemographic and clinical characteristics (Supplemental Table 1) and in physical function, fatigue, and pain (Table 2). Survivors diagnosed with cardiovascular disease or lung disease were more likely to have been diagnosed with lung cancer. Survivors with arthritis were significantly more likely to be women (57.3\%) and have early stage cancer (76.5\%) compared to survivors without arthritis. Survivors diagnosed with diabetes were more likely to have less than a high school education (28.4\%) compared to survivors without diabetes (19.3\%).

After controlling for sociodemographic characteristics, clinical characteristics, and history of other comorbid conditions, cancer survivors with CVD reported lower physical functioning ( $\beta=-3.85, p<0.001$; Table 3$)$, higher pain $(\beta=2.74, p<0.001$; Table 3$)$, and higher fatigue $(\beta=3.45$, $p<0.001$; Table 3) compared to survivors without CVD. The adjusted means for fatigue reached the threshold for a minimally important difference between survivors with CVD and those without CVD (Table 2).

After controlling for all other characteristics, cancer survivors with lung disease had significantly lower physical functioning $(\beta=-4.96, p<0.001$; Table 3$)$ and higher levels of pain $(\beta=2.45, p<0.001$; Table 3$)$ and fatigue $(\beta=4.49$, $p<0.001$; Table 3 ) compared to survivors without lung disease. The adjusted means for both physical function and fatigue reached the threshold for a minimally important difference between survivors with lung disease and those without lung disease (Table 2).

After controlling for all other characteristics, cancer survivors with diabetes had significantly worse physical functioning $(\beta=-2.05, p<0.001$; Table 3$)$ and higher levels of pain $(\beta=1.93, p<0.001$; Table 3$)$ and fatigue $(\beta=1.87$ $p<0.001$; Table 3 ) compared to survivors without diabetes. None of the adjusted means reached the threshold for minimally important differences between survivors with and without diabetes (Table 2).

After controlling for sociodemographic characteristics, clinical characteristics, and history of other comorbid conditions, cancer survivors with arthritis had significantly worse physical function $(\beta=-3.32, p<0.001$; Table 3$)$ and higher levels of pain $(\beta=4.34, p<0.001$; Table 3$)$ and fatigue $(\beta=2.57, p<0.001$; Table 3$)$ compared to survivors without arthritis. The adjusted means for pain and fatigue reached the threshold for a minimally important difference between survivors with arthritis and those without arthritis (Table 2).

Cancer survivors with 1 comorbid condition reported significantly worse physical functioning $(\beta=-3.24$, $p<0.001)$, higher pain $(\beta=3.11, p<0.001)$, and higher fatigue $(\beta=2.72, p<0.001)$ compared to survivors with only a cancer history (Table 4). Cancer survivors with a history of 2 or more comorbid conditions reported even worse physical functioning $(\beta=-7.63, p<0.001)$, higher pain $(\beta=7.27$, $p<0.001)$, and higher fatigue $(\beta=7.25, p<0.001)$ compared to survivors with only a cancer history. For both physical function and pain, the adjusted means reached the threshold for a minimally important difference between cancer only and cancer +2 or more and between cancer +1 and cancer +2 or more. The fatigue adjusted means reached the threshold for a minimally important difference between all groups. The results of our sensitivity analysis are reported in Supplemental Table 1. Overall, these findings found that the direction of the associations (worse physical functioning and higher symptoms among survivors with more comorbidities) was consistent across cancer types and with the non-stratified models.

\section{Discussion}

Our study found that three-quarters of older cancer survivors were multimorbid, which is similar to findings in the broader Medicare population. Specifically, 63\% of Medicare beneficiaries $65-74$ years report multimorbidity, and $78 \%$ of Medicare beneficiaries 75-84 years report multimorbidity [1]. Older survivors with arthritis reported clinically relevant pain and fatigue, survivors with lung disease reported clinically relevant physical function limitations and fatigue, survivors with CVD reported clinically relevant fatigue, and survivors with diabetes did not report any clinically relevant symptoms or functioning concerns, consistent with prior literature [1]. Lung disease has previously been found to be associated with impaired physical function and increased total symptom burden [34, 35]. Similarly, prior work has found that diabetes is not as strongly associated with HRQOL compared to other comorbid conditions [36, 37]. A strength of our study is the utilization of populationbased cancer registries (SEER) to identify and recruit the sample, providing a more generalizable estimate of the multimorbidity burden and HRQOL of older cancer survivors. Overall, our study results suggest that understanding older cancer survivor's multimorbidity profile may be clinically important to mitigate potentially adverse outcomes following a cancer diagnosis.

We also compared associations between specific comorbid conditions and the total burden of multimorbidity on HRQOL. We found that the presence of any single comorbid condition (cancer +1 condition) had a lower symptom burden than a comorbid condition with an identified HRQOL deficit. For example, pain reported by older cancer survivors with arthritis was higher than for cancer survivors reporting any comorbid condition. These findings are consistent with existing evidence that identified stronger associations between certain comorbid conditions and specific outcomes [28].

Previous research has also indicated that having multiple comorbid conditions is associated with lower HRQOL 
Table 3 Generalized linear models examining each selected comorbidity and the PROMIS domains

\begin{tabular}{|c|c|c|c|c|c|c|c|c|}
\hline & \multicolumn{2}{|c|}{ Cardiovascular disease } & \multicolumn{2}{|l|}{ Arthritis } & \multicolumn{2}{|l|}{ Diabetes } & \multicolumn{2}{|l|}{ Lung disease } \\
\hline & $B(\mathrm{SE})$ & $p$ value & $B(\mathrm{SE})$ & $p$ value & $B(\mathrm{SE})$ & $p$ value & $B(\mathrm{SE})$ & $p$ value \\
\hline \multicolumn{9}{|l|}{ Physical function } \\
\hline \multicolumn{9}{|c|}{ History of selected condition $(\mathrm{ref}=\mathrm{no})$} \\
\hline Yes & $-3.68(0.58)$ & $<0.001$ & $-3.16(0.40)$ & $<0.001$ & $-2.08(0.43)$ & $<0.001$ & $-5.10(0.57)$ & $<0.001$ \\
\hline \multicolumn{9}{|c|}{ Other comorbid conditions $(\mathrm{ref}=\mathrm{no})$} \\
\hline Yes & $-5.33(0.43)$ & $<0.001$ & $-4.62(0.40)$ & $<0.001$ & $-5.34(0.41)$ & $<0.001$ & $-4.78(0.42)$ & $<0.001$ \\
\hline Age at diagnosis & $-0.23(0.58)$ & $<0.001$ & $-0.24(0.04)$ & $<0.001$ & $-0.24(0.04)$ & $<0.001$ & $-0.24(0.04)$ & $<0.001$ \\
\hline \multicolumn{9}{|l|}{ Sex $($ ref $=$ male $)$} \\
\hline Female & $-1.68(0.58)$ & 0.004 & $-1.38(0.58)$ & 0.016 & $-1.20(0.58)$ & 0.039 & $-1.58(0.57)$ & 0.006 \\
\hline \multicolumn{9}{|c|}{ Race/ethnicity (ref=non-Hispanic White) } \\
\hline Non-Hispanic Black & $-0.95(0.56)$ & 0.091 & $-0.93(0.56)$ & 0.098 & $-0.95(0.57)$ & 0.094 & $-1.08(0.56)$ & 0.054 \\
\hline Hispanic & $-1.59(0.59)$ & 0.007 & $-1.42(0.59)$ & 0.016 & $-1.58(0.60)$ & 0.008 & $-1.86(0.59)$ & 0.002 \\
\hline Non-Hispanic Asian & $-1.24(0.59)$ & 0.037 & $-1.35(0.59)$ & 0.022 & $-1.37(0.60)$ & 0.022 & $-1.55(0.59)$ & 0.009 \\
\hline Other race/multiple races & $-0.88(1.21)$ & 0.465 & $-0.96(1.20)$ & 0.422 & $-1.04(1.21)$ & 0.386 & $-0.98(1.20)$ & 0.413 \\
\hline \multicolumn{9}{|c|}{$\begin{array}{l}\text { Education (ref }=\text { completed college degree } \\
\text { or higher) }\end{array}$} \\
\hline$<$ High school (HS) grad & $-3.57(0.60)$ & $<0.001$ & $-3.57(0.60)$ & $<0.001$ & $-3.82(0.60)$ & $<0.001$ & $-3.59(0.60)$ & $<0.001$ \\
\hline HS grad or some college & $-1.66(0.47)$ & $<0.001$ & $-1.73(0.47)$ & $<0.001$ & $-1.93(0.47)$ & $<0.001$ & $-1.77(0.47)$ & $<0.001$ \\
\hline \multicolumn{9}{|c|}{ Marital status (ref $=$ married/partnered) } \\
\hline Not partnered* & $-0.87(0.42)$ & .042 & $-0.73(0.42)$ & 0.083 & $-0.85(0.42)$ & 0.045 & $-0.79(0.42)$ & 0.061 \\
\hline \multicolumn{9}{|l|}{ Cancer site $($ ref $=$ prostate $)$} \\
\hline Breast & $-2.42(0.86)$ & 0.005 & $-2.40(0.85)$ & 0.005 & $-2.62(0.86)$ & 0.002 & $-2.37(0.85)$ & 0.006 \\
\hline Lung & $-5.06(0.70)$ & $<0.001$ & $-5.24(0.69)$ & $<0.001$ & $-5.32(0.69)$ & $<0.001$ & $-4.11(0.72)$ & $<0.001$ \\
\hline Colorectal & $-2.59(0.73)$ & $<0.001$ & $-2.85(0.73)$ & $<0.001$ & $-2.81(0.73)$ & $<0.001$ & $-2.44(0.73)$ & $<0.001$ \\
\hline Non-Hodgkin lymphoma & $-1.59(0.85)$ & 0.062 & $-1.77(0.84)$ & 0.036 & $-1.95(0.85)$ & 0.022 & $-1.46(0.84)$ & 0.083 \\
\hline Gynecologic & $-3.22(1.08)$ & 0.003 & $-3.20(1.08)$ & 0.003 & $-3.35(1.08)$ & 0.002 & & \\
\hline \multicolumn{9}{|l|}{ Chemotherapy $(\mathrm{ref}=\mathrm{no})$} \\
\hline Yes & $-2.83(0.48)$ & $<0.001$ & $-2.93(0.48)$ & $<0.001$ & $-2.90(0.48)$ & $<0.001$ & $-2.85(0.48)$ & $<0.001$ \\
\hline \multicolumn{9}{|l|}{ Radiation $($ ref $=$ no $)$} \\
\hline Yes & $-0.72(0.42)$ & 0.090 & $-0.73(0.42)$ & 0.082 & $-0.70(0.42)$ & 0.098 & $-0.57(0.42)$ & 0.177 \\
\hline \multicolumn{9}{|l|}{ Surgery $($ ref $=$ no $)$} \\
\hline Yes & $0.68(0.49)$ & 0.164 & $0.68(0.48)$ & 0.159 & $0.70(0.49)$ & 0.149 & $0.70(0.48)$ & 0.145 \\
\hline \multicolumn{9}{|l|}{ Stage (ref $=$ early stage) } \\
\hline Late stage & $-1.57(0.51)$ & 0.002 & $-1.58(0.51)$ & 0.002 & $-1.57(0.51)$ & 0.002 & $-1.58(0.51)$ & 0.002 \\
\hline \multicolumn{9}{|l|}{ Pain } \\
\hline \multicolumn{9}{|c|}{ History of selected condition $(\mathrm{ref}=\mathrm{no})$} \\
\hline Yes & $2.83(0.67)$ & $<0.001$ & $4.32(0.46)$ & $<0.001$ & $1.97(0.50)$ & $<0.001$ & $2.39(0.66)$ & $<0.001$ \\
\hline \multicolumn{9}{|c|}{ Other comorbid conditions $(\mathrm{ref}=\mathrm{no})$} \\
\hline Yes & $5.29(0.50)$ & $<0.001$ & $3.77(0.46)$ & $<0.001$ & $5.05(0.47)$ & $<0.001$ & $5.34(0.49)$ & $<0.001$ \\
\hline Age at diagnosis & $-0.08(0.05)$ & 0.091 & $-0.07(0.05)$ & 0.098 & $-0.06(0.05)$ & 0.172 & $-0.07(0.05)$ & 0.108 \\
\hline \multicolumn{9}{|l|}{ Sex $($ ref $=$ male $)$} \\
\hline Female & $0.32(0.67)$ & 0.635 & $-0.06(0.66)$ & 0.927 & $-0.07(0.67)$ & 0.913 & $0.16(0.67)$ & 0.807 \\
\hline Race/ethnicity $(\mathrm{ref}=$ non $-\mathrm{Hi}$ & & & & & & & & \\
\hline Non-Hispanic Black & $1.56(0.65)$ & 0.016 & $1.50(0.64)$ & 0.019 & $1.55(0.65)$ & 0.018 & $1.58(0.65)$ & 0.015 \\
\hline Hispanic & $2.58(0.69)$ & $<0.001$ & $2.47(0.68)$ & $<0.001$ & $2.57(0.69)$ & $<0.001$ & $2.65(0.69)$ & $<0.001$ \\
\hline Non-Hispanic Asian & $3.01(0.68)$ & $<0.001$ & $3.15(0.67)$ & $<0.001$ & $3.14(0.69)$ & $<0.001$ & $3.09(0.68)$ & $<0.001$ \\
\hline Other race/multiple races & $4.68(1.38)$ & .001 & $4.86(1.36)$ & $<0.001$ & $4.90(1.38)$ & $<0.001$ & $4.72(1.38)$ & 0.001 \\
\hline $\begin{array}{l}\text { Education (ref }=\text { completed } \\
\text { or higher) }\end{array}$ & & & & & & & & \\
\hline$<$ High School (HS) grad & $4.01(0.69)$ & $<0.001$ & $3.95(0.68)$ & $<0.001$ & $4.23(0.69)$ & $<0.001$ & $4.12(0.69)$ & $<0.001$ \\
\hline
\end{tabular}


Table 3 (continued)

\begin{tabular}{|c|c|c|c|c|c|c|c|c|}
\hline & Cardiovascular & $r$ disease & Arthritis & & Diabetes & & Lung disease & \\
\hline & $B(\mathrm{SE})$ & $p$ value & $B(\mathrm{SE})$ & $p$ value & $B(\mathrm{SE})$ & $p$ value & $B(\mathrm{SE})$ & $p$ value \\
\hline HS grad or some college & $2.10(0.54)$ & $<0.001$ & $2.14(0.53)$ & $<0.001$ & $2.32(0.54)$ & $<0.001$ & $2.25(0.54)$ & $<0.001$ \\
\hline Marital status (ref $=$ married & & & & & & & & \\
\hline Not partnered* & $0.82(0.49)$ & 0.093 & $0.73(0.48)$ & 0.131 & $0.81(0.49)$ & 0.098 & $0.78(0.49)$ & 0.112 \\
\hline Cancer site $($ ref $=$ prostate $)$ & & & & & & & & \\
\hline Breast & $1.18(0.99)$ & 0.235 & $1.09(0.98)$ & 0.264 & $1.35(0.99)$ & 0.175 & $1.22(0.99)$ & 0.218 \\
\hline Lung & $2.08(0.80)$ & 0.010 & $2.34(0.79)$ & 0.003 & $2.29(0.80)$ & 0.004 & $1.97(0.83)$ & 0.018 \\
\hline Colorectal & $0.92(0.84)$ & 0.274 & $1.34(0.83)$ & 0.108 & $1.09(0.84)$ & 0.195 & $0.98(0.84)$ & 0.244 \\
\hline Non-Hodgkin lymphoma & $0.41(0.98)$ & 0.674 & $0.60(0.96)$ & 0.535 & $0.73(0.98)$ & 0.457 & $0.40(0.98)$ & 0.681 \\
\hline Gynecologic & $1.32(1.25)$ & 0.290 & $1.33(1.23)$ & 0.279 & $1.43(1.25)$ & 0.252 & $1.41(1.25)$ & 0.258 \\
\hline Chemotherapy $(\mathrm{ref}=\mathrm{no})$ & & & & & & & & \\
\hline Yes & $2.33(0.55)$ & $<0.001$ & $2.40(0.55)$ & $<0.001$ & $2.39(0.55)$ & $<0.001$ & $2.35(0.55)$ & $<0.001$ \\
\hline Radiation $(\mathrm{ref}=\mathrm{no})$ & & & & & & & & \\
\hline Yes & $1.00(0.49)$ & 0.040 & $1.11(0.48)$ & 0.021 & $1.00(0.49)$ & 0.040 & $0.97(0.49)$ & 0.048 \\
\hline Surgery $(\mathrm{ref}=\mathrm{no})$ & & & & & & & & \\
\hline Yes & $0.34(0.56)$ & 0.540 & $0.34(0.55)$ & 0.535 & $0.33(0.56)$ & 0.557 & $0.31(0.56)$ & 0.577 \\
\hline Stage (ref $=$ early stage) & & & & & & & & \\
\hline Late stage & $1.28(0.59)$ & 0.030 & $1.43(0.58)$ & 0.014 & $1.29(0.59)$ & 0.028 & $1.27(0.59)$ & 0.031 \\
\hline Fatigue* & & & & & & & & \\
\hline History of selected conditior & & & & & & & & \\
\hline Yes & $3.42(0.66)$ & $<0.001$ & $2.51(0.45)$ & $<0.001$ & $1.91(0.49)$ & $<0.001$ & $4.56(0.65)$ & $<0.001$ \\
\hline Other comorbid conditions & & & & & & & & \\
\hline Yes & $4.85(0.49)$ & $<0.001$ & $4.74(0.45)$ & $<0.001$ & $4.81(0.47)$ & $<0.001$ & $4.24(0.48)$ & $<0.001$ \\
\hline Age at diagnosis & $0.03(0.04)$ & 0.471 & $0.05(0.04)$ & 0.282 & $0.05(0.04)$ & 0.278 & $0.04(0.04)$ & 0.314 \\
\hline Sex $($ ref $=$ male $)$ & & & & & & & & \\
\hline Female & $1.31(0.66)$ & 0.046 & $1.10(0.65)$ & 0.912 & $0.87(0.66)$ & 0.187 & $1.21(0.65)$ & 0.064 \\
\hline Race/ethnicity $(\mathrm{ref}=$ non-Hi & & & & & & & & \\
\hline Non-Hispanic Black & $-0.96(0.64)$ & 0.134 & $-0.98(0.63)$ & 0.122 & $-0.97(0.64)$ & 0.133 & $-0.84(0.64)$ & 0.186 \\
\hline Hispanic & $-0.14(0.68)$ & 0.839 & $-0.34(0.67)$ & 0.615 & $-0.15(0.68)$ & 0.824 & $0.10(0.67)$ & 0.882 \\
\hline Non-Hispanic Asian & $0.11(0.67)$ & 0.865 & $0.23(0.67)$ & 0.727 & $0.24(0.68)$ & 0.720 & $0.39(0.67)$ & 0.566 \\
\hline Other race/multiple races & $2.51(1.35)$ & 0.064 & $2.56(1.34)$ & 0.057 & $2.72(1.36)$ & 0.045 & $2.68(1.35)$ & 0.048 \\
\hline $\begin{array}{l}\text { Education }(\mathrm{ref}=\text { completed } \\
\text { or higher) }\end{array}$ & & & & & & & & \\
\hline$<$ High school (HS) grad & $3.11(0.68)$ & $<0.001$ & & & $2.25(0.68)$ & $<0.001$ & $3.14(0.68)$ & $<0.001$ \\
\hline HS grad or some college & $1.81(0.53)$ & 0.001 & $1.85(0.53)$ & 0.001 & $2.07(0.53)$ & $<0.001$ & $1.92(0.53)$ & $<0.001$ \\
\hline Marital status $(\mathrm{ref}=$ married & & & & & & & & \\
\hline Not partnered* & $0.90(0.48)$ & 0.062 & $0.74(0.48)$ & 0.121 & $0.89(0.48)$ & 0.065 & $0.84(0.48)$ & 0.079 \\
\hline Cancer site $($ ref $=$ prostate $)$ & & & & & & & & \\
\hline Breast & $0.71(0.97)$ & 0.465 & $0.66(0.97)$ & 0.494 & $0.92(0.98)$ & 0.345 & $0.70(0.97)$ & 0.469 \\
\hline Lung & $3.65(0.79)$ & $<0.001$ & $3.70(0.78)$ & $<0.001$ & $3.92(0.79)$ & $<0.001$ & $2.83(0.82)$ & 0.001 \\
\hline Colorectal & $1.78(0.83)$ & 0.032 & $1.94(0.82)$ & 0.019 & $2.00(0.83)$ & 0.016 & $1.66(0.83)$ & 0.045 \\
\hline Non-Hodgkin lymphoma & $1.43(0.96)$ & 0.137 & $1.57(0.96)$ & 0.100 & $1.78(0.96)$ & 0.064 & $1.34(0.96)$ & 0.163 \\
\hline Gynecologic & $1.66(1.23)$ & 0.175 & $1.60(1.22)$ & 0.189 & & & $1.75(1.22)$ & 0.153 \\
\hline Chemotherapy $(\mathrm{ref}=\mathrm{no})$ & & & & & & & & \\
\hline Yes & $3.34(0.55)$ & $<0.001$ & $3.43(0.54)$ & $<0.001$ & $3.40(0.55)$ & $<0.001$ & $3.35(0.54)$ & $<0.001$ \\
\hline Radiation $($ ref $=$ no) & & & & & & & & \\
\hline Yes & $1.79(0.48)$ & $<0.001$ & $1.77(0.48)$ & $<0.001$ & $1.77(0.48)$ & $<0.001$ & $1.65(0.48)$ & 0.001 \\
\hline Surgery $(\mathrm{ref}=\mathrm{no})$ & & & & & & & & \\
\hline Yes & $-0.58(0.55)$ & 0.290 & $-0.59(0.54)$ & 0.283 & $-0.61(0.55)$ & 0.265 & $-0.62(0.55)$ & 0.260 \\
\hline
\end{tabular}


Table 3 (continued)

\begin{tabular}{|c|c|c|c|c|c|c|c|c|}
\hline & \multicolumn{2}{|c|}{ Cardiovascular disease } & \multicolumn{2}{|l|}{ Arthritis } & \multicolumn{2}{|l|}{ Diabetes } & \multicolumn{2}{|l|}{ Lung disease } \\
\hline & $B(\mathrm{SE})$ & $p$ value & $B(\mathrm{SE})$ & $p$ value & $B(\mathrm{SE})$ & $p$ value & $B(\mathrm{SE})$ & $p$ value \\
\hline \multicolumn{9}{|c|}{ Stage (ref $=$ early stage) } \\
\hline Late stage & $2.13(0.58)$ & $<0.001$ & $2.10(0.57)$ & $<0.001$ & $2.12(0.58)$ & $<0.001$ & $2.13(0.58)$ & $<0.001$ \\
\hline
\end{tabular}

*Not partnered marital status includes: never married, divorced, widowed, or separated

Table 4 Generalized linear models examining total number of comorbid conditions and PROMIS domains

\begin{tabular}{|c|c|c|c|c|c|c|}
\hline & \multicolumn{2}{|c|}{ Physical function } & \multicolumn{2}{|l|}{ Pain } & \multicolumn{2}{|l|}{ Fatigue } \\
\hline & $B(\mathrm{SE})$ & $p$ value & $B(\mathrm{SE})$ & $p$ value & $B(\mathrm{SE})$ & $p$ value \\
\hline \multicolumn{7}{|c|}{ Comorbid conditions $(r e f=0)$} \\
\hline 1 condition & $-3.08(0.50)$ & $<0.001$ & $3.24(0.58)$ & $<0.001$ & $2.55(0.57)$ & $<0.001$ \\
\hline $2+$ condition & $-7.52(0.47)$ & $<0.001$ & $7.20(0.54)$ & $<0.001$ & $7.03(0.53)$ & $<0.001$ \\
\hline Age at diagnosis & $-0.25(0.04)$ & $<0.001$ & $-0.06(0.05)$ & 0.177 & $0.05(0.04)$ & 0.242 \\
\hline \multicolumn{7}{|l|}{$\operatorname{Sex}(r e f=$ male $)$} \\
\hline Female & $-1.44(0.57)$ & 0.011 & $0.15(0.66)$ & 0.821 & $1.10(0.65)$ & 0.090 \\
\hline \multicolumn{7}{|c|}{ Racelethnicity (ref=non-Hispanic White) } \\
\hline Non-Hispanic Black & $-1.01(0.56)$ & 0.070 & $1.60(0.64)$ & 0.013 & $-0.91(0.63)$ & 0.149 \\
\hline Hispanic & $-1.54(0.59)$ & 0.009 & $2.52(0.68)$ & $<0.001$ & $-0.21(0.67)$ & 0.754 \\
\hline Non-Hispanic Asian & $-1.47(0.59)$ & 0.012 & $3.22(0.68)$ & $<0.001$ & $0.35(0.66)$ & 0.593 \\
\hline Other race/multiple races & $-1.07(1.19)$ & 0.368 & $4.87(1.36)$ & $<0.001$ & $2.75(1.34)$ & 0.042 \\
\hline \multicolumn{7}{|c|}{ Education (ref = completed college degree or higher) } \\
\hline$<$ High school (HS) grad & $-3.68(0.59)$ & $<0.001$ & $4.09(0.69)$ & $<0.001$ & $3.21(0.67)$ & $<0.001$ \\
\hline HS grad or some college & $-1.85(0.46)$ & $<0.001$ & $2.24(0.53)$ & $<0.001$ & $1.98(0.52)$ & $<0.001$ \\
\hline \multicolumn{7}{|c|}{ Marital status $($ ref $=$ married/partnered $)$} \\
\hline Not partnered ${ }^{\mathrm{a}}$ & $-0.64(0.42)$ & 0.130 & $0.62(0.49)$ & 0.205 & $0.66(0.48)$ & 0.164 \\
\hline \multicolumn{7}{|l|}{ Cancer site $($ ref $=$ prostate $)$} \\
\hline Breast & $-2.27(0.85)$ & 0.008 & $1.01(0.98)$ & 0.304 & $0.54(0.96)$ & 0.577 \\
\hline Lung & $-4.93(0.69)$ & $<0.001$ & $1.92(0.79)$ & 0.016 & $3.49(0.78)$ & $<0.001$ \\
\hline Colorectal & $-2.60(0.72)$ & $<0.001$ & $0.91(0.83)$ & 0.274 & $1.78(0.82)$ & 0.030 \\
\hline Non-Hodgkin lymphoma & $-1.65(0.84)$ & 0.049 & $0.45(0.97)$ & 0.645 & $1.48(0.95)$ & 0.119 \\
\hline Gynecologic & $-3.03(1.07)$ & 0.005 & $1.13(1.24)$ & 0.359 & $1.46(1.21)$ & 0.227 \\
\hline \multicolumn{7}{|l|}{ Chemotherapy $(r e f=n o)$} \\
\hline Yes & $-3.01(0.48)$ & $<0.001$ & $2.48(0.55)$ & $<0.001$ & $3.52(0.54)$ & $<0.001$ \\
\hline \multicolumn{7}{|l|}{ Radiation $($ ref $=n o)$} \\
\hline Yes & $-0.66(0.42)$ & 0.115 & $0.96(0.48)$ & 0.047 & $1.74(0.47)$ & $<0.001$ \\
\hline \multicolumn{7}{|l|}{ Surgery $(r e f=n o)$} \\
\hline Yes & $0.68(0.48)$ & 0.154 & $0.34(0.55)$ & 0.533 & $-0.59(0.54)$ & 0.279 \\
\hline \multicolumn{7}{|l|}{ Stage $(r e f=$ early stage $)$} \\
\hline Late stage & $-1.56(0.50)$ & 0.002 & $1.29(0.58)$ & 0.027 & $2.13(0.57)$ & $<0.001$ \\
\hline
\end{tabular}

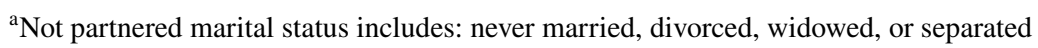

among older cancer survivors and may be associated with lower overall survival [34-39]. Our results support previous research, indicating that cumulative multimorbidity burden may be associated with lower HRQOL, regardless of the specific chronic conditions. Older cancer survivors with two or more additional comorbid conditions reported worse pain, fatigue, and greater physical function limitations compared to survivors reporting only one comorbidity and survivors without any additional comorbidities. The current COVID19 pandemic has highlighted both risks associated with having underlying health conditions [40], and challenges with getting needed care, given stay-at-home orders and social distancing guidelines. For cancer patients with multimorbidity, such challenges are even greater. The healthcare 
workforce, primarily within primary care and oncology, is already experiencing problems in providing timely, highquality care to all cancer survivors [41]. As a result, it is critical to find ways to identify those patients who may have greater symptom burden and functional decline, to both support ways to help patients manage at home and make referrals to appropriate care when needed. Consideration of various potential risk factors for poor outcomes, including the presence of other chronic conditions, is a necessary step to identify older survivors who may need more regular monitoring and assistance in symptom management as they transition to survivorship.

Our findings should be interpreted considering the study limitations. First, the survey was a cross-sectional assessment of health outcomes 6 to 12 months after a cancer diagnosis. This precludes our ability to examine the predictive nature of multimorbidity on health outcomes after cancer, and the patterns of associations between multimorbidity and HRQOL may look different in survivors further away from diagnosis. Additionally, we do not know when each of these conditions was diagnosed in relation to survivors' cancer diagnoses and treatment; this timing may be important [42]. We also do not have information on the severity or longevity of each of these conditions and thus are unable to indicate the true level of multimorbidity burden [43]. Beyond the timing of diagnosis and severity of the comorbidities, there may also be additional missing variables (e.g. geographic region, annual income) that could affect the associations presented in this study. Finally, although the sample used in the current study was recruited through the population-based SEER cancer registries, we only utilized four of the SEER registries. This may limit the generalizability of our study findings to the broader population of older cancer survivors in the US.

The transition from active treatment to post-treatment survivorship is a critical point in the care of cancer survivors and includes the management of the physical and psychosocial effects of cancer and its treatment $[44,45]$. Our results complement and extend a growing interest in survivorship research-risk stratification of survivorship care [41]. Riskstratified care involves identifying those patients who have the greatest needs and then 'triaging' patients to different care pathways based on their level of risk. Patients with the highest risk (for example, determined by the type and count of comorbidity, prognosis/risk of recurrence, late effects and/or ability to self-manage) could continue their followup with oncology providers, while individuals at moderate or low risk could be seen by advanced practice providers, primary care or both [41]. Examining select comorbid conditions may identify survivors at risk for specific poor outcomes (e.g. arthritis and pain), but examining the total number of conditions may identify survivors at risk for multiple poor outcomes. Recognizing the key role of multimorbidity in the health of older cancer survivors will aid in the timely identification of problematic symptoms and the introduction of personalized and appropriate strategies for prevention or management.

Author contributions Study design and data collection were performed by Arnold Potosky and Roxanne Jensen. Data analysis were performed by Elizabeth Siembida, Roxanne Jensen, and Ashley Wilder Smith. The first draft of the manuscript was written by Elizabeth Siembida, and all authors commented on previous versions of the manuscript. All authors read and approved the final manuscript.

Funding Research reported in this publication was supported by the National Institutes of Health under award number U01 AR057971 (M-PIs: Potosky, Moinpour).

Data availability De-identified data from this publication can be made available upon written request to the corresponding author.

\section{Compliance with ethical standards}

Conflict of interest The authors have no conflicts of interest to declare.

Ethical approval All study procedures that involved human participants were in accordance with the ethical standards of the 1964 Helsinki declaration and its later amendments or comparable ethical standards. The study procedures were approved by the Institutional Review Board at Georgetown University.

Informed consent Informed consent was obtained from all individual participants included in the study.

\section{References}

1. Centers for Medicare and Medicaid Services. (2012). Chronic conditions among Medicare beneficiaries. Chartbook. Baltimore, MD.

2. Huang, I., Hudson, M. M., Robison, L. L., \& Krull, K. R. (2017). Differential impact of symptom prevalence and chronic conditions on quality of life in cancer survivors and non-cancer individuals: A population study. Cancer Epidemiology, Biomarkers \& Prevention, 26, 1124-1132.

3. Ng, H. S., Roder, D., Koczwara, B., \& Vitry, A. (2018). Comorbidity, physical and mental health among cancer patients and survivors: An Australian population-based study. Asia-Pacific Journal of Clinical Oncology, 14, e181-e192.

4. Yamagishi, A., Morita, T., Miyashita, M., \& Kimura, F. (2009). Symptom prevalence and longitudinal follow-up in cancer outpatients receiving chemotherapy. Journal of Pain and Symptom Management, 37, 823-830.

5. Brant, J. M., Beck, S. L., Dudley, W. N., Cobb, P., Pepper, G., \& Miaskowski, C. (2011). Symptom trajectories during chemotherapy in outpatients with lung cancer, colorectal cancer, or lymphoma. European Journal of Oncology Nursing, 15, 470-477.

6. Freak-Poli, R., Ikram, M. A., Franco, O. H., Hofman, A., \& Tiemeier, H. (2018). Depressive symptoms prior to and after incident cardiovascular disease and long-term survival: A populationbased study of older persons. Depression \& Anxiety, 35, 18-31. 
7. Servaes, P., Gielissen, M. F. M., Verhagen, S., \& Bleijenberg, G. (2007). The course of severe fatigue in disease-free breast cancer patients: A longitudinal study. Psycho-Oncology, 16, 787-795.

8. Wang, X. S., Zhao, F., Fisch, M. J., O’Mara, A. M., Cella, D., Mendoza, T. R., et al. (2014). Prevalence and characteristics of moderate to severe fatigue: A multicenter study in cancer patients and survivors. Cancer, 120, 425-432.

9. Pandya, C., Magnuson, A., Flannery, M., Zittel, J., Duberstein, P., Loh, K. P., et al. (2019). Association between symptom burden and physical function in older patients with cancer. Journal of the American Geriatrics Society, 67, 998-1004.

10. Laroche, F., Perrot, S., Medkour, T., Cottu, P., Plerga, J., Beerblock, K., et al. (2017). Quality of life and impact of pain in women treated with aromatase inhibitors for breast cancer: A multicenter cohort study. PLoS ONE, 12(1), e0187165.

11. Curt, G. A., Breitbart, W., Cella, D., Groopman, J. E., Horning, S. J., Itri, L. M., et al. (2000). Impact of cancer-related fatigue on the lives of patients: New findings from the fatigue coalition. The Oncologist, 5, 353-360.

12. Fleming, S. T., Sabatino, S. A., Kimmick, G., Cress, R., Wu, X., Trentham-Dietz, A., et al. (2011). Developing a claim-based version of the ACE-27 comorbidity index: A comparison with medical record review. Medical Care, 49, 752-760.

13. Elixhauser, A., Steiner, C., Harris, D. R., \& Coffey, R. M. (1998). Comorbidity measures for use with administrative data. Medical Care, 36(1), 8-27.

14. Charlson, M. E., Pompei, P., Ales, K. L., \& MacKenzie, C. R. (1987). A new method of classifying prognostic comorbidity in longitudinal studies: Development and validation. Journal of Chronic Disease, 40, 373-383.

15. Klabunde, C. N., Legler, J. M., Warren, J. L., Baldwin, L., \& Schrag, D. (2007). A refined comorbidity measurement algorithm for claims-based studies of breast, prostate, colorectal, and lung cancer patients. Annals of Epidemiology, 17, 584-590.

16. Koroukian, S. M., Bakaki, P. M., Schluchter, M. D., \& Owusu, C. (2011). Treatment and survival patterns in relation to multimorbidity in patients with locoregional breast and colorectal cancer. Journal of Geriatric Oncology, 2(3), 200-208.

17. Koroukian, S. M., Xu, F., Bakaki, P. M., Diaz-Insua, M., Towe, T. P., \& Owusu, C. (2010). Comorbidities, functional limitations, and geriatric syndromes in relation to treatment and survival patterns among elders with colorectal cancer. Journal of Gerontology: Medical Sciences, 65A(3), 322-329.

18. Ording, A. G., Boffetta, P., Garne, J. P., Nyström, P. M. W., Cronin-Fenton, D., Frøslev, T., et al. (2015). Relative mortality rates from incident chronic diseases among breast cancer survivors - A 14 year follow-up of five-year survivors diagnosed in Denmark between 1994 and 2007. European Journal of Cancer, 51, 767-775.

19. Aarts, M. J., Aerts, J. F., van den Borne, B. E., Biesma, B., Lemmens, V. E. P. P., \& Kloover, J. S. (2015). Comorbidity in patients with small-cell lung cancer: Trends and prognostic impact. Clinical Lung Cancer, 16(4), 282-291.

20. Sarfati, D., Koczwara, B., \& Jackson, C. (2016). The impact of comorbidity on cancer and its treatment. CA: A Cancer Journal for Clinicians, 66, 337-350.

21. Edwards, B. K., Noone, A., Mariotto, A. B., Simard, E. P., Boscoe, F. P., Henley, S. J., et al. (2014). Annual report to the nation on the status of cancer, 1975-2010, featuring prevalence of comorbidity and impact on survival among persons with lung, colorectal, breast, or prostate cancer. Cancer, 120, 1290-1314.

22. Nilsson, J., Berglund, A., Bergström, S., Bergqvist, M., \& Lambe, M. (2017). The role of comorbidity in the management and prognosis in nonsmall cell lung cancer: A population-based study. Acta Oncologica, 56(7), 949-956.
23. Edwards, M. J., Campbell, I. D., Lawrenson, R. A., \& KuperHommel, M. J. (2017). Influence of comorbidity on chemotherapy use for early breast cancer: A systematic review and meta-analysis. Breast Cancer Research and Treatment, 165, 17-39.

24. Chang, H., Shih, S., \& Lin, F. (2012). Impact of comorbidities on the outcomes of older patients receiving rectal cancer surgery. International Journal of Gerontology, 6, 285-289.

25. Wollschläger, D., Meng, X., Wöckel, A., Janni, W., Kreienberg, R., Blettner, M., et al. (2018). Comorbidity-dependent adherence to guidelines and survival in breast cancer - Is there a role for guideline adherence in comorbid breast cancer patients? A retrospective study with 2137 patients. The Breast Journal, 24, $120-127$.

26. Sabatino, S. A., Thompson, T. D., Wu, X., Fleming, S. T., Kimmick, G. G., Trentham-Dietz, A., et al. (2014). The influence of diabetes severity on receipt of guideline-concordant treatment for breast cancer. Breast Cancer Research and Treatment, 146, 199-209.

27. Kimmick, G., Fleming, S. T., Sabatino, S. A., Wu, X., Hwang, W., Wilson, J. F., et al. (2014). Comorbidity burden and guidelineconcordant care for breast cancer. Journal of American Geriatrics Society, 62, 482-488.

28. Smith, A. W., Reeve, B. B., Bellizzi, K. M., Harlan, L. C., Klabunde, C. N., Amsellem, M., et al. (2008). Cancer, comorbidities, and health-related quality of life of older adults. Health Care Financing Review, 29(4), 41-56.

29. Portz, J. D., Kutner, J. S., Blatchford, P. J., \& Ritchie, C. S. (2017). High symptom burden and low functional status in the setting of multimorbidity. Journal of American Geriatrics Society, 65, 2285-2289.

30. Davis, L. E., Bubis, L. D., Mahar, A. L., Li, Q., Sussman, J., Moody, L., et al. (2018). Patient-reported symptoms after breast cancer diagnosis and treatment: A retrospective cohort study. European Journal of Cancer, 101, 1-11.

31. Jensen, R. E., Moinpour, C. M., Keegan, T. H. M., Cress, R. D., Wu, X., Paddock, L. E., et al. (2016). The measuring your health study: Leveraging community-based cancer registry recruitment to establish a large, diverse cohort of cancer survivors for analyses of measurement equivalence and validity of the Patient Reported Outcomes Measurement Information System ${ }^{\circledR}$ (PROMIS ${ }^{\circledR}$ ) short form items. Psychological Test and Assessment Modeling, 58(1), 99-117.

32. Cella, D., Riley, W., Stone, A., Rothrock, N., Reeve, B., Yount, S., et al. (2010). The Patient-Reported Outcomes Measurement Information System (PROMIS) developed and tested its first wave of adult self-reported health outcome item banks: 2005-2008. Journal of Clinical Epidemiology, 63, 1179-1194.

33. Yost, K. J., Eton, D. T., Garcia, S. F., \& Cella, D. (2011). Minimally important differences were estimated for six PatientReported Outcomes Measurement Information System-Cancer scales in advanced stage cancer patients. Journal of Clinical Epidemiology, 64, 507-516.

34. Cummings, A., Grimmett, C., Calman, L., Patel, M., Permyakova, N. V., Winter, J., et al. (2018). Comorbidities are associated with poorer quality of life and functioning and worse symptoms in the 5 years following colorectal cancer surgery: Results from the ColoREctal Well-being (CREW) cohort study. Psycho-Oncology, 27, 2427-2435.

35. Yi, Y., Ban, W. H., \& Sohng, K. (2018). Effect of COPD on symptoms, quality of life and prognosis in patients with advanced nonsmall cell lung cancer. BMC Cancer, 18, 1053.

36. Wang, J., Sun, L., Li, J., Cong, X., Chen, X., Tang, Z., et al. (2015). Comorbid chronic diseases and their associations with quality of life among gynecological cancer survivors. BMC Public Health, 15, 965. 
37. Wang, J., Sun, L., Ding, N., Li, J., Gong, X., Chen, X., et al. (2016). The association between comorbidities and the quality of life among colorectal cancer survivors in the People's Republic of China. Patient Preference and Adherence, 10, 1071-1077.

38. Boakye, D., Rillmann, B., Walter, V., Jansen, L., Hoffmeister, M., \& Brenner, H. (2018). Impact of comorbidity and frailty on prognosis in colorectal cancer patients: A systematic review and meta-analysis. Cancer Treatment Reviews, 64, 30-39.

39. Williams, G. R., Deal, A. M., Lund, J. L., Chang, Y., Muss, H. B., Pergolotti, M., et al. (2017). Patient-reported comorbidity and survival in older adults with cancer. The Oncologist, 22, 1-7.

40. Centers for Disease Control and Prevention. (2020). People with certain medical conditions. Retrieved August 11, 2020, from https ://www.cdc.gov/coronavirus/2019-ncov/need-extra-precautions/ people-with-medical-conditions.html.

41. Mayer, D. K., \& Alfano, C. M. (2019). Personalized risk-stratified cancer follow-up care: Its potential for healthier survivors, happier clinicians and lower costs. Journal of National Cancer Institute, $11,442-448$.

42. Shao, S., Gill, A. A., Zahm, S. H., Jatoi, I., Shriver, C. D., McGlynn, K. A., et al. (2017). Diabetes and overall survival among breast cancer patients in the U.S. military health system. Cancer Epidemiology, Biomarkers \& Prevention, 27, 50-57.

43. Austin, S. R., Wong, Y., Uzzo, R. G., Beck, J. R., \& Egleston, B. L. (2015). Why summary comorbidity measures such as the Charlson comorbidity index and Elixhauser score work. Medical Care, 53, e65-e72.

44. Yabroff, K. R., Gansler, T., Wender, R. C., Cullen, K. J., \& Brawley, O. W. (2019). Minimizing the burden of cancer in the United States: Goals for a high-performing health care system. $C A$ : A Cancer Journal for Clinicians, 69, 166-183.

45. Naylor, M., \& Berlinger, N. (2016). Transitional care: A priority for health care organizational ethics. Hastings Center Report, 46, S39-S42.

Publisher's Note Springer Nature remains neutral with regard to jurisdictional claims in published maps and institutional affiliations.

\section{Affiliations}

\section{Elizabeth J. Siembida ${ }^{1,2,5}$ (i) $\cdot$ Ashley Wilder Smith ${ }^{3} \cdot$ Arnold L. Potosky $^{4} \cdot$ Kristi D. Graves $^{4} \cdot$ Roxanne E. Jensen $^{3}$}

1 Division of Cancer Control and Population Sciences, National Cancer Institute, Rockville, MD, USA

2 Cancer Prevention Fellowship Program, National Cancer Institute, Rockville, MD, USA

3 Outcomes Research Branch, National Cancer Institute, Rockville, MD, USA
4 Cancer Prevention and Control Program, Lombardi Comprehensive Cancer Center, Washington, DC, USA

5 Center for Health Innovation and Outcomes Research, Feinstein Institute for Medical Research, Northwell Health, 600 Community Drive, Suite 403, Manhasset, NY 11030, USA 